\title{
Renormalisation of composite operators in lattice QCD: perturbative versus nonperturbative
}

\section{Göckeler ${ }^{* a}$, R. Horsley ${ }^{b}$, Y. Nakamura ${ }^{a}$, H. Perlt ${ }^{c}$, D. Pleiter ${ }^{d}$, P.E.L. Rakow ${ }^{e}$,} A. Schäfer ${ }^{a}$, G. Schierholz ${ }^{a f}$, A. Schiller ${ }^{c}$, H. Stüben ${ }^{g}$ and J.M. Zanotti ${ }^{b}$

${ }^{a}$ Institut für Theoretische Physik, Universität Regensburg, 93040 Regensburg, Germany

${ }^{b}$ School of Physics and Astronomy, University of Edinburgh, Edinburgh EH9 3JZ, UK

${ }^{c}$ Institut für Theoretische Physik, Universität Leipzig, 04109 Leipzig, Germany

${ }^{d}$ Deutsches Elektronen-Synchrotron DESY and John von Neumann-Institut für Computing NIC, 15738 Zeuthen, Germany

${ }^{e}$ Theoretical Physics Division, Department of Mathematical Sciences, University of Liverpool, Liverpool L69 3BX, UK

${ }^{f}$ Deutsches Elektronen-Synchrotron DESY, 22603 Hamburg, Germany

${ }^{g}$ Konrad-Zuse-Zentrum für Informationstechnik Berlin, 14195 Berlin, Germany

E-mail: meinulf.goeckeler@physik.uni-regensburg.de

\section{QCDSF/UKQCD Collaboration}

\begin{abstract}
The perturbative and nonperturbative renormalisation of quark-antiquark operators in lattice QCD with two flavours of clover fermions is investigated within the research programme of the QCDSF collaboration. Operators with up to three derivatives are considered. The nonperturbative results based on the RI-MOM scheme are compared with estimates from one- and two-loop lattice perturbation theory.
\end{abstract}

The XXVIII International Symposium on Lattice Field Theory, Lattice2010

June 14-19, 2010

Villasimius, Italy

\footnotetext{
* Speaker.
} 


\section{Introduction}

If one uses a lattice regulator to define $\mathrm{QCD}$, renormalisation means that bare parameters, i.e., the coupling constant and the quark masses, acquire a dependence on the lattice spacing $a$ such that physical observables like hadron masses have a finite continuum limit $a \rightarrow 0$. This should then guarantee that all physical quantities have a well-defined continuum limit.

However, it is not always possible to calculate the observables of interest directly on the lattice. Quite often one can only evaluate certain matrix elements of composite operators from which the desired physical quantity is derived in a second step. In general, it is then necessary to introduce $a$-dependent renormalisation factors $Z$ for these operators in order to get a finite continuum limit. For example, moments of structure functions in deep-inelastic scattering are physical observables, but they can only be calculated as products of a (perturbatively calculated) Wilson coefficient and a hadronic matrix element of a local composite operator, which is a long-distance quantity to be computed on the lattice.

It is therefore important to investigate the renormalisation of composite operators in lattice QCD, and the present contribution describes some of the efforts the QCDSF collaboration has undertaken in this field. For the details we refer to Ref. [1].

\section{How to evaluate renormalisation factors on the lattice}

In principle it is possible to calculate the $Z$ factors of multiplicatively renormalisable operators by lattice perturbation theory (for a review see Ref. [2]). However, perturbation theory on the lattice is computationally much more complex than in the continuum and therefore the calculations rarely extend beyond one-loop order (see, however, Refs. [3, 4, 5, 6]). Moreover, lattice perturbation theory usually converges rather slowly so that the accuracy of perturbative renormalisation factors is limited, even if some improvement scheme is applied.

Therefore nonperturbative approaches have been developed, in particular methods based on the Schrödinger functional (for reviews see Ref. [7]) and the RI-MOM scheme [8]. It is the latter approach in the slightly modified form of the $\mathrm{RI}^{\prime}$ - MOM scheme that was adopted to produce the nonperturbative results presented below, where we consider only composite operators constructed from two quark fields, Dirac matrices and a few covariant derivatives in between.

In the RI' - MOM scheme the basic objects are quark two-point functions with an insertion of the operator under consideration at momentum zero. A suitable renormalisation condition is imposed, which does not rely on a particular regularisation so that it can be applied on the lattice as well as in the continuum. The latter property entails the possibility to use continuum perturbation theory in order to calculate conversion factors leading from the operators renormalised in the $\mathrm{RI}^{\prime}$ - MOM scheme to operators renormalised in, e.g., the $\overline{\mathrm{MS}}$ scheme.

For a multiplicatively renormalisable operator we express the operator renormalised in the scheme $\mathscr{S}$ at the renormalisation scale $\mu$ as $Z_{\text {bare }}^{\mathscr{S}}(\mu) \mathscr{O}(a)$, where $\mathscr{O}(a)$ denotes the bare operator on the lattice and the $a$ dependence of the renormalisation factor has been suppressed. For scales 
satisfying $\Lambda_{\mathrm{QCD}}^{2} \ll \mu^{2} \ll 1 / a^{2}$ the quantity

$$
Z^{\mathrm{RGI}}=\left(2 \beta_{0} \frac{g^{\mathscr{S}}(\mu)^{2}}{16 \pi^{2}}\right)^{-\gamma_{0} /\left(2 \beta_{0}\right)} \exp \left\{\int_{0}^{g^{\mathscr{S}}(\mu)} \mathrm{d} g\left(\frac{\gamma^{\mathscr{S}}(g)}{\beta^{\mathscr{S}}(g)}+\frac{\gamma_{0}}{\beta_{0} g}\right)\right\} Z_{\text {bare }}^{\mathscr{S}}(\mu)=\Delta Z^{\mathscr{S}}(\mu) Z_{\text {bare }}^{\mathscr{S}}(\mu)
$$

is independent of the scale and the scheme. Here $g^{\mathscr{S}}(\mu)$ denotes the renormalised coupling in the scheme $\mathscr{S}$, while $\gamma^{\mathscr{S}}$ and $\beta^{\mathscr{S}}$ are the anomalous dimension and the beta function, respectively, whose one-loop coefficients are $\gamma_{0}$ and $\beta_{0}$. In the simulations we compute $Z_{\mathrm{bare}}^{\mathrm{RI}^{\prime}-\mathrm{MOM}}$ and evaluate

$$
Z^{\mathrm{RGI}}=\Delta Z^{\mathscr{S}}(\mu) Z_{\mathrm{RI}^{\prime}-\mathrm{MOM}}^{\mathscr{S}}(\mu) Z_{\mathrm{bare}}^{\mathrm{RI}^{\prime}-\mathrm{MOM}}(\mu) .
$$

This expression would be identical to $(2.1)$ if we knew $\Delta Z^{\mathscr{S}}$ and the conversion factor $Z_{\mathrm{RI}^{\prime}-\mathrm{MOM}}^{\mathscr{S}}$ exactly. However, these quantities are calculated in continuum perturbation theory, so they come with certain truncation errors and the result will depend on the choice of the intermediate scheme $\mathscr{S}$. What will also matter is the expansion parameter. It turns out to be advantageous not to use $g^{\overline{\mathrm{MS}}}$ but the coupling $g^{\widehat{\mathrm{MOM} g g}}$ as defined in Ref. [9].

In bare lattice perturbation theory one-loop results for renormalisation factors are of the form

$$
Z_{\text {bare }}^{\mathscr{S}}(\mu)_{\text {pert }}=1-\frac{g^{2}}{16 \pi^{2}}\left(\gamma_{0} \ln (a \mu)+\Delta\right)+O\left(g^{4}\right)
$$

As this expansion in the bare lattice coupling $g$ is often poorly convergent, tadpole-improved perturbation theory has been invented, where for an operator with $n_{D}$ covariant derivatives one has

$$
Z_{\text {bare }}^{\mathscr{S}}(\mu)_{\mathrm{ti}}=u_{0}^{1-n_{D}}\left[1-\frac{g_{\square}^{2}}{16 \pi^{2}}\left(\gamma_{0} \ln (a \mu)+\Delta+\left(n_{D}-1\right) \frac{4}{3} \pi^{2}\right)+O\left(g^{4}\right)\right] .
$$

Here the fourth root of the average plaquette $u_{0}=\left\langle\frac{1}{3} \operatorname{tr} U_{\square}\right\rangle^{1 / 4}$ is taken from the simulations and the expansion parameter is the boosted coupling $g_{\square}^{2}=g^{2} / u_{0}^{4}$. Combining renormalisation group improvement with tadpole improvement one arrives at "tadpole-improved renormalisation-groupimproved boosted perturbation theory" or TRB perturbation theory for short [1].

In the above expressions for $Z$ derived from lattice perturbation theory lattice artefacts have been neglected and only the logarithmic $a$ dependence has been kept. This is only justified if $a^{2} \mu^{2} \ll 1$. Unfortunately, this condition is not always fulfilled in our simulations. On the other hand, it is straightforward (though increasingly involved for more complicated operators) to do calculations in one-loop lattice perturbation theory with arbitrary values of $a^{2} \mu^{2}$. One can then use the difference between the $Z s$ with and without lattice artefacts to correct the nonperturbative simulation results for discretisation errors of $O\left(g^{2}\right)$.

\section{The simulations}

We use gauge field configurations generated by the QCDSF-UKQCD collaborations with two degenerate flavours of clover fermions and the Wilson plaquette action for the gauge field. Configurations for 4 values of $\beta$ are at our disposal, $\beta=5.20,5.25,5.29,5.40$, corresponding to lattice spacings $a \approx 0.086,0.079,0.075,0.067 \mathrm{fm}$. To set the scale for the lattice spacing we have taken the value $r_{0}=0.467 \mathrm{fm}$ for the Sommer parameter, and we use $r_{0} \Lambda_{\overline{\mathrm{MS}}}=0.617$ [10] when the $\Lambda$ 

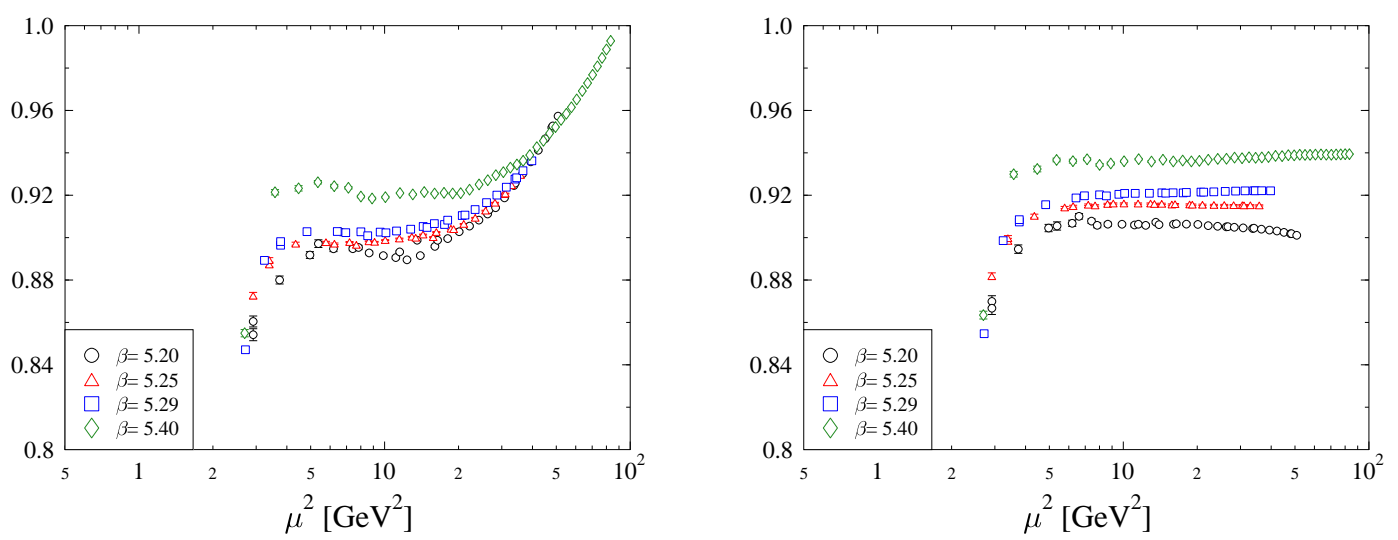

Figure 1: $Z^{\mathrm{RGI}}$ for the tensor current $\bar{\psi} \sigma_{\mu \nu} \psi$ before (left panel) and after (right panel) the perturbative subtraction of lattice artefacts.

parameter of QCD is needed. For each $\beta$ we have between three and five sea quark masses so that a chiral extrapolation to vanishing quark mass is possible.

As mentioned above, we have to compute quark two-point functions with an insertion of the operator under consideration, i.e., three-point functions. We restrict ourselves to flavour-nonsinglet operators so that only quark-line connected contributions must be evaluated. This is conveniently done with the help of momentum sources introduced in Ref. [11].

\section{Extracting the renormalisation factors}

The simplest procedure for obtaining a value of $Z^{\mathrm{RGI}}$ would be to plot the right-hand side of Eq. (2.2), i.e., $\Delta Z^{\mathscr{S}}(\mu) Z_{\mathrm{RI}^{\prime}-\mathrm{MOM}}^{\mathscr{S}}(\mu) Z_{\mathrm{bare}}^{\mathrm{RI}^{\prime} \mathrm{MOM}}(\mu)$ versus $\mu$ and to fit a constant to these data in an interval of $\mu$ where they form a plateau. Examples of such plots before and after the perturbative subtraction of lattice artefacts are shown in Fig. 1. Equivalently one could fit the values obtained for $Z_{\mathrm{bare}}^{\mathscr{S}}(\mu)=Z_{\mathrm{RI}^{\prime}-\mathrm{MOM}}^{\mathscr{S}}(\mu) Z_{\mathrm{bare}}^{\mathrm{RI}^{\prime}-\mathrm{MOM}}(\mu)$ in the plateau region by $\Delta Z^{\mathscr{S}}(\mu)^{-1} Z^{\mathrm{RGI}}$ (with $Z^{\mathrm{RGI}}$ as fit parameter).

However, there are two effects that jeopardize the reliability of this approach: lattice artefacts, which show up at large values of $\mu$ and truncation errors of the perturbative expansions in $\Delta Z^{\mathscr{S}}$ and $Z_{\mathrm{RI}^{\prime}-\mathrm{MOM}}^{\mathscr{S}}$, which become noticeable at small values of $\mu$. They might even conspire to produce a fake plateau. Therefore we have tried to incorporate higher terms in the perturbative series treating the corresponding coefficients as additional fit parameters. Similarly we have attempted to correct for discretisation effects by including a simple ansatz for lattice artefacts. Again, the parameters in this ansatz have to be fitted. We fit the data for all four $\beta$ values simultaneously and only the quantities $Z^{\mathrm{RGI}}$, our final results, depend on $\beta$, the other parameters do not. Two examples of such fits are shown in Fig. 目. For further details concerning the fit procedure we must refer to Ref. [1]. The results determined by this fit procedure will be called fit results in the following.

Unfortunately, these fits work only for the perturbatively subtracted data. However, for technical reasons, we have applied our subtraction only for operators with at most one covariant derivative. Hence we must apply a different procedure for operators with more than one derivative: We 

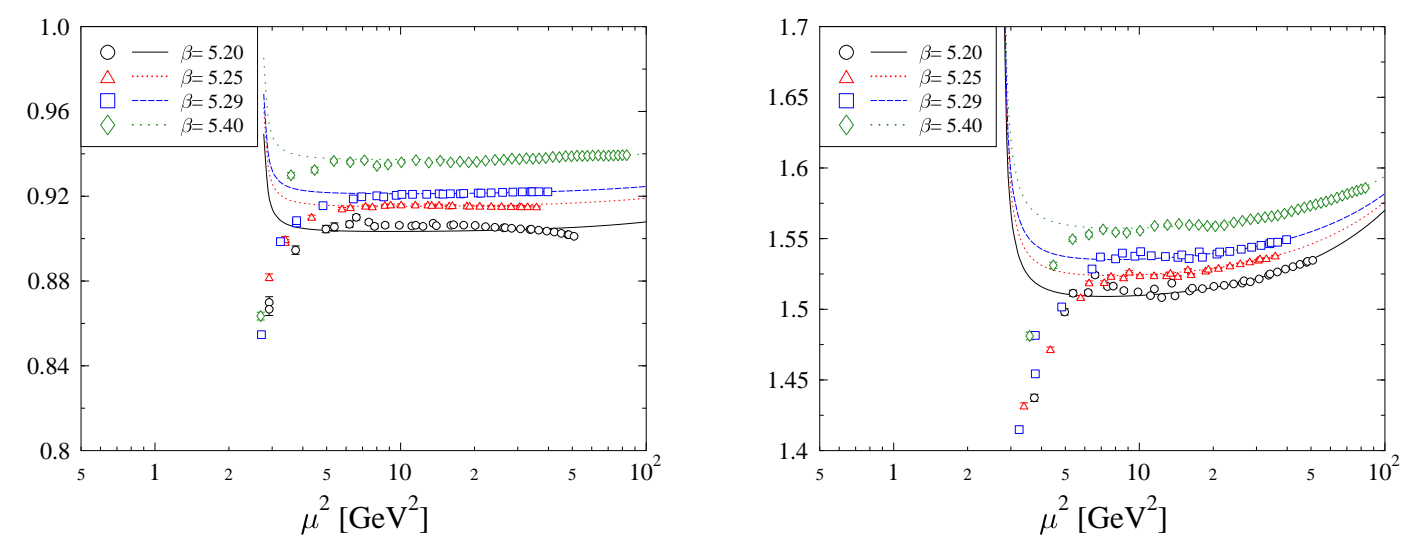

Figure 2: $Z^{\text {RGI }}$ (perturbatively subtracted) for the tensor current (left panel) and $\bar{\psi} \gamma_{\{1} \stackrel{\leftrightarrow}{D}_{4\}} \psi$ (right panel) as a function of the renormalisation scale. Also shown are the fit curves used for the determination of $Z^{\text {RGI }}$.

read off $Z^{\mathrm{RGI}}$ at $\mu^{2}=20 \mathrm{GeV}^{2}$ and take as the error the maximum of the differences with the results at $\mu^{2}=10 \mathrm{GeV}^{2}$ and $\mu^{2}=30 \mathrm{GeV}^{2}$. These results will be called interpolation results in the following. Obviously, this method can also be applied to subtracted data. The errors assigned by the fits appear to be seriously underestimated since they are mainly determined by the statistical uncertainties. Therefore it seems to be more reasonable to finally use only the errors from the interpolation method as these take into account also some of the systematic effects.

\section{Results}

Due to lack of space we have to restrict the presentation of our results to operators without derivatives, i.e. the "currents"

$$
\mathscr{O}^{S}=\bar{u} d, \mathscr{O}^{P}=\bar{u} \gamma_{5} d, \mathscr{O}_{\mu}^{V}=\bar{u} \gamma_{\mu} d, \mathscr{O}_{\mu}^{A}=\bar{u} \gamma_{\mu} \gamma_{5} d, \mathscr{O}_{\mu \nu}^{T}=\bar{u} \sigma_{\mu \nu} d
$$

and the quark wave function renormalisation constant $Z_{q}$.

In Fig. 3 we plot the results at $\beta=5.40$ extracted from the perturbatively subtracted data, both by interpolation and by means of the fit procedure, and the interpolation results based on the unsubtracted numbers as well as one-loop perturbative estimates. Ideally the nonperturbative results should agree within the errors. In reality, this is not always true. Note, however, that the errors of the fit results only account for the (rather small) statistical uncertainties of the raw data while the errors of the interpolation results are dominated by systematic effects. The one-loop perturbative estimates are larger than the nonperturbative values, but tadpole improvement works. TRB perturbation theory, on the other hand, leads to further improvement only in a few cases, for some operators it is even worse than ordinary tadpole-improved perturbation theory.

In Fig. 母 we compare our fit results for the operators without derivatives with the one-loop and two-loop perturbative estimates, again for $\beta=5.40$. The numbers from bare lattice perturbation theory, represented by circles in the figure, exhibit the expected behavior: The two-loop results come closer to the nonperturbative numbers than the one-loop estimates, though only slightly in 


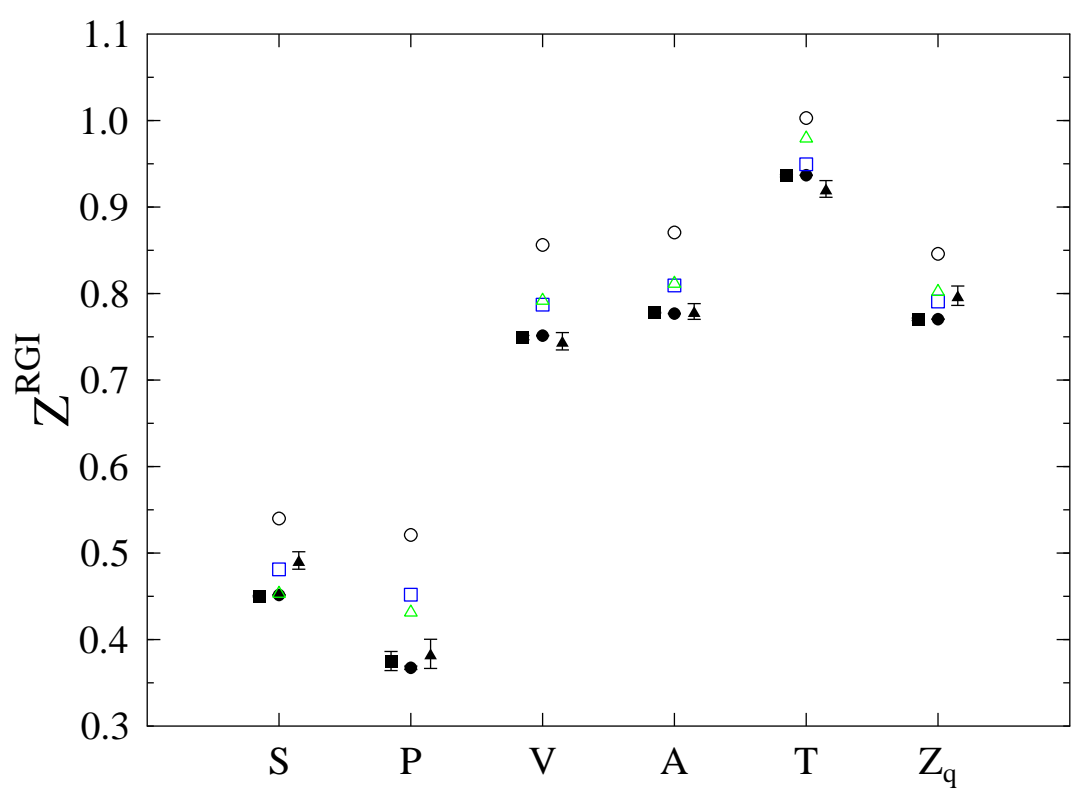

Figure 3: Results for operators without derivatives at $\beta=5.40$. The filled symbols correspond to our fit results (circles), interpolation results based on subtracted (squares) and unsubtracted (triangles) data. The open symbols represent estimates from bare perturbation theory (circles), tadpole-improved perturbation theory (squares) and TRB perturbation theory (triangles) based on one-loop calculations.

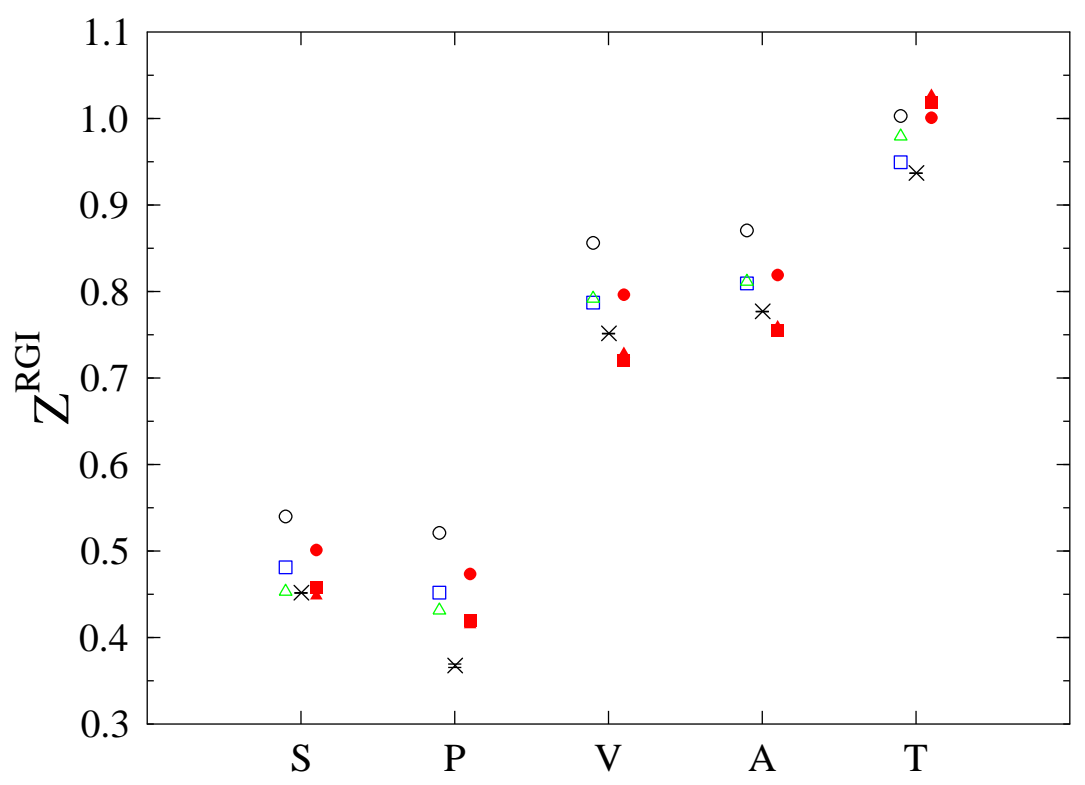

Figure 4: Results for operators without derivatives at $\beta=5.40$ compared with one- and two-loop lattice perturbation theory. The crosses correspond to our nonperturbative results obtained by fits of the subtracted data. The open symbols represent estimates from bare perturbation theory (circles), tadpole-improved perturbation theory (squares) and TRB perturbation theory (triangles) in the one-loop approximation. The corresponding estimates based on two-loop calculations are shown by the filled symbols. 
the case of the tensor current. Except for the tensor current, tadpole improvement works also in the two-loop approximation moving the perturbative values closer to the nonperturbative numbers. However, the results from TRB perturbation theory, shown by triangles, do not differ much from the values found by tadpole improved two-loop perturbation theory.

\section{Concluding remarks}

The $\mathrm{RI}^{\prime}$ - MOM scheme has been established as a method for nonperturbative renormalisation that can (relatively) easily be implemented for arbitrary lattice fermions. Momentum sources allow us to deal with all operators in a single simulation and at the same time to achieve small statistical errors, but the required computer time is proportional to the number of momenta considered. One of the largest sources of systematic uncertainties are discretisation effects: Here the perturbative subtraction of lattice artefacts has proved very helpful. Continuum perturbation theory is needed for the conversion to the $\overline{\mathrm{MS}}$ scheme. Obviously, one should use as many loops as are available, but additional improvements are possible through the careful choice of intermediate schemes and expansion parameters. Still, it seems that the available perturbative results cannot describe the scale dependence below the (surprisingly large) value of about $5 \mathrm{GeV}^{2}$, as seen, e.g., in Fig. 1. Remarkably enough, there are now renormalisation factors calculated in two-loop lattice perturbation theory. Unfortunately, it is difficult to predict their accuracy without comparing with nonperturbative results, but (at least in most cases) improvement seems to work.

\section{Acknowledgements}

The numerical calculations have been performed on the apeNEXT and APEmille computers at NIC/DESY (Zeuthen). This work has been supported in part by the EU Integrated Infrastructure Initiative HadronPhysics2 and by the DFG (SFB/TR55 "Hadron Physics from Lattice QCD").

\section{References}

[1] M. Göckeler et al., arXiv:1003.5756 [hep-lat].

[2] S. Capitani, Phys. Rept. 382, 113 (2003) [arXiv:hep-lat/0211036].

[3] Q. Mason, H. Trottier and R. Horgan, PoS LAT2005, 011 (2006) [arXiv:hep-lat/0510053].

[4] A. Skouroupathis and H. Panagopoulos, Phys. Rev. D 76, 094514 (2007) [Erratum-ibid. D 78, 119901 (2008)] [arXiv:0707.2906 [hep-lat]].

[5] A. Skouroupathis and H. Panagopoulos, Phys. Rev. D 79, 094508 (2009) [arXiv:0811.4264 [hep-lat]].

[6] F. Di Renzo, V. Miccio, L. Scorzato and C. Torrero, PoS LAT2006, 156 (2006) [arXiv:hep-lat/0609077].

[7] R. Sommer, arXiv:hep-ph/9711243; arXiv:hep-lat/0611020.

[8] G. Martinelli et al., Nucl. Phys. B 445, 81 (1995) [arXiv:hep-lat/9411010].

[9] K.G. Chetyrkin and A. Rétey, arXiv:hep-ph/0007088.

[10] M. Göckeler et al., Phys. Rev. D 73, 014513 (2006) [arXiv:hep-ph/0502212].

[11] M. Göckeler et al., Nucl. Phys. B 544, 699 (1999) [arXiv:hep-lat/9807044]. 